\title{
Self-gated Radial MRI for Respiratory Motion Compensation on Hybrid PET/MR Systems
}

\author{
Robert Grimm ${ }^{1}$, Sebastian Fürst ${ }^{2}$, Isabel Dregely ${ }^{2}$, Christoph Forman ${ }^{1}$, \\ Jana Maria Hutter ${ }^{1}$, Sibylle I. Ziegler ${ }^{2}$, Stephan Nekolla ${ }^{2}$, Berthold Kiefer ${ }^{3}$, \\ Markus Schwaiger ${ }^{2}$, Joachim Hornegger ${ }^{1}$, and Tobias Block ${ }^{4}$ \\ 1 Pattern Recognition Lab, FAU Erlangen, Erlangen, Germany \\ 2 Department of Nuclear Medicine, TU Munich, Munich, Germany \\ 3 Siemens Healthcare MR, Erlangen, Germany \\ 4 Department of Radiology, NYU Langone Medical Center, New York City, NY, USA
}

\begin{abstract}
Accurate localization and uptake quantification of lesions in the chest and abdomen using PET imaging is challenging due to the respiratory motion during the exam. The advent of hybrid PET/MR systems offers new ways to compensate for respiratory motion without exposing the patient to additional radiation. The use of self-gated reconstructions of a $3 \mathrm{D}$ radial stack-of-stars GRE acquisition is proposed to derive a high-resolution MRI motion model. The self-gating signal is used to perform respiratory binning of the simultaneously acquired PET raw data. Matching $\mu$-maps are generated for every bin, and post-reconstruction registration is performed in order to obtain a motion-compensated PET volume from the individual gates. The proposed method is demonstrated in-vivo for three clinical patients. Motioncorrected reconstructions are compared against ungated and gated PET reconstructions. In all cases, motion-induced blurring of lesions in the liver and lung was substantially reduced, without compromising SNR as it is the case for gated reconstructions.
\end{abstract}

\section{Introduction}

With scan times of between two and ten minutes per bed position, positron emission tomography (PET) imaging of the lung and abdomen is affected by respiratory motion. Breath-hold techniques cannot be applied for such long time, and respiratory gating approaches lack clinical acceptance because they reduce the scan efficiency and, thus, lead to increased noise or excessive scan time. Clinical PET scans are affected by two types of artifacts due to respiratory motion: First, the mismatch between the MR-based attenuation correction map ( $\mu$-map) used for PET reconstruction, which is typically acquired in an endexpiratory breathhold, and the PET image itself, which is acquired during free breathing, causes an under- or overestimation of tracer activity especially in the vicinity of the diaphragm [1]. Second, respiration leads to local image blurring (smearing) along the direction of motion, i.e., primarily in the cranio-caudal direction. This can result in an incorrectly estimated volume, shape, and uptake of lesions [2-4] as well as in reduced conspicuity especially of small lesions. 
Respiration is a predominantly periodic type of motion that can be compensated for if a model of the motion is available. Typically, this model consists of a displacement vector field describing the nonrigid deformation that maps voxels between different respiratory states. A comprehensive review of respiratory motion models has recently been published by McClelland et al. [5].

In hybrid PET/MR scanners, respiratory motion models can be formed either by near-realtime 3D MR sequences 6-8] or by retrospective gating and averaging over multiple respiratory cycles [4, 9]. Retrospectively gated MR motion models can further be subdivided into three categories according to the acquisition method: 1) The displacement fields can be measured directly using tagged MRI 9 -11], 2) the volume can be sampled using a 2D multi-slice technique [4, 7] or 3) using a 3D acquisition [6, 7].

According to these characteristic features, the proposed approach for respiratory motion compensation on integrated PET/MR scanners can be classified as follows. It utilizes a 3D radial stack-of-stars MRI sequence. This sequence allows for self-gating, i.e. a respiratory signal can be derived without the need for additional MR navigator echoes or sensors attached to the patient. Moreover, the trajectory allows for retrospective gating, without having to face discontinuities between slices that affect 2D multi-slice methods. The model is acquired for the whole PET acquisition and thereby assures high spatial fidelity and can adapt to changes in the respiratory pattern.

After derivation of the MR-based motion model, it is utilized for motioncorrected PET image reconstruction. The PET gates are reconstructed using conventional algorithms, then warped to a common respiratory phase, and finally averaged [4, 6]. We followed this approach rather than incorporating the motion model directly into the PET reconstruction, as recently suggested in literature [7, 12 14], because we focused on the formation of the motion model in this work. The utilized post-reconstruction registration approach is well-studied, reproducible, and provides comparable results given that the respiratory bins have similar and sufficient count statistics [13 15].

\section{Methods}

Our approach is based on the following scan protocol for a combined whole-body $\mathrm{PET} / \mathrm{MR}$ scanner. First, the $\mu$-map is acquired conventionally $(20 \mathrm{~s}$ breathhold scan at end-expiration). Afterwards, MR and PET data are acquired simultaneously. Detailed sequence parameters can be found in Section 4 .

In the subsequent data processing chain, described in more detail in the following sections, a motion model is computed from the MR data. It is subsequently applied to deform the $\mu$-map to different respiratory states. A self-gating signal extracted from the MR scan is used to perform respiratory gated PET reconstructions at different levels of inspiration, each utilizing the matching $\mu$ map. Finally, the inverse deformations are used to co-register and combine the individual PET volumes. 


\subsection{Self-gated Radial MRI}

The respiratory motion model is generated with the help of a T1-weighted 3D radial stack-of-stars spoiled gradient echo sequence with fat suppression. A golden-angle increment of $111.25^{\circ}$ between subsequently acquired radial angles distributes the sampling incoherently but approximately uniformly [16] over the readout plane, facilitating retrospectively gated reconstruction.

This particular k-space trajectory, shown in Fig. 1, allows to derive a self-gating signal (SGS) from the central k-space partition $k_{z}=0$ that is sampled every $N_{z} \cdot$ TR [17]. Each sample of the self-gating signal is computed as the mean absolute value of the central three $\mathrm{k}$-space samples in a readout. A suitable coil element to derive the signal is determined automatically by computing the Fourier transform of the SGS for every coil and selecting the one with the highest ratio of the peak in

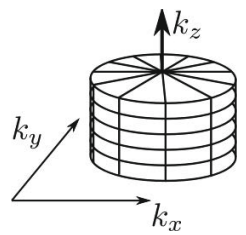

Fig. 1. Radial stack-of-stars the range of expected respiratory frequencies to the energy in the remaining high-frequency coefficients. It should be noted that the SGS is of qualitative nature and, unlike a 1D navigator echo or projection-based self-gating [6], does not indicate an absolute displacement e.g. of the liver.

Variable amplitude-based binning is applied to partition the radial readouts into $N_{\text {Bins }}$ bins containing equal amounts of data, according to the respective self-gating signal amplitude. This scheme ensures comparable statistics also for the PET listmode data that were gated in the same manner (see Section 2.3).

\subsection{Motion Modeling}

A state-of-the art nonrigid registration algorithm that was recently proposed for lung registration [18] is employed to compute the deformation between the respiratory-gated MR volumes. Its output is a $3 \mathrm{D}$ deformation field $\boldsymbol{T}_{i, j}$ that maps a volume at respiratory phase $i$ to a volume at respiratory phase $j$. The deformations are estimated from each respiratory phase to the reference phase 1 (end-exhale), and vice versa.

\subsection{Motion-Compensated PET Reconstruction}

To generate matching $\mu$-maps $\hat{M}_{i}$ for the respiratory phases $i \in\left\{2 \ldots N_{\text {Bins }}\right\}$, the original end-exhale $\mu$-map $M_{1}$ is warped to each respiratory state using the corresponding deformation field $\boldsymbol{T}_{1, i}$.

The self-gating signal from the MRI acquisition is used to reconstruct gated images from the PET listmode data. For this purpose, each listmode event is assigned to the same bin as the closest SGS sample according to the respective timestamps. Static (ungated) images are reconstructed from all available PET data as well. The vendor-provided clinical reconstruction algorithm (orderedsubset expectation maximization, OSEM) is applied. The respective $\mu$-maps (warped / native) are utilized for attenuation correction. After reconstruction, the gated PET volumes $P_{i}$ are co-registered to the end-expiratory state by applying $\boldsymbol{T}_{i, 1}$. Finally, the co-registered images are averaged. 


\section{$3 \quad$ Experiments}

\subsection{Self-gating}

To study the accuracy of the respiratory self-gating, images were reconstructed using $N_{\text {Bins }} \in\{2 \ldots 15\}$ respiratory bins. Partitioning the data into more bins reduces the SNR in every bin but allows more accurate separation of different respiratory states. A virtual 1D navigator column (averaged over $3 \times 3$ voxels in the transversal plane) was extracted at the apex of the liver dome in the reconstructed volumes. The position of the liver edge at each respiratory state was detected by Canny filtering $\left(\sigma=1.5, \theta_{\text {low }}=0.8, \theta_{\text {high }}=0.95\right)$ along the column.

\subsection{PET Reconstruction}

The three datasets were analyzed visually as well as quantitatively, in terms of apparent lesion volume that was computed by placing ellipsoidal VOIs using syngo TrueD (Siemens Healthcare, Erlangen, Germany) and applying a fixed threshold for segmentation. The following reconstructions were compared:

1. $R_{\text {All }}$ : Ungated reconstruction using all PET data and original $\mu$-map.

2. $R_{40}$ : Gold standard: Gated reconstruction using the $40 \%$ of the data with the least variation in the SGS amplitude.

To examine the effects of post-reconstruction registration, the gated PET images were computed with the adapted $\mu$-map and warped to the reference state before fusion. This gives the corrected reconstruction:

3. $G_{5}$ : Gating to 5 bins, warped $\mu$-maps, post-reconstruction registration.

\section{Clinical Study}

The protocol described in Section 2 was used for three oncological patient studies $P_{1}, P_{2}, P_{3}$, conducted on a 3 Tesla hybrid PET/MR system (Biograph mMR; Siemens Healthcare, Erlangen, Germany). Written consent from the subjects and approval from the local ethics committee was obtained prior to the examinations. Between 401 and $455 \mathrm{MBq}$ of ${ }^{18} \mathrm{~F}$-FDG were administered as radionuclide agent, 96 - 148 min before the study. The $\mu$-maps were acquired with a 3D Dixon GRE sequence while the patients were instructed to hold their breath at endexpiration. No particular respiratory instruction was given for the following PET listmode acquisition, during which the motion modeling scan was carried out: $P_{1}(\mathrm{f} / 58 \mathrm{y} / 82 \mathrm{~kg})$ : coronal slab orientation (FOV $450 \times 450 \times 245 \mathrm{~mm}^{3}$ ), spatial resolution $1.6 \times 1.6 \times 1.7 \mathrm{~mm}^{3}$, 288 pixel matrix, 145 slices $(50 \%$ slice resolution, 5/8 partial Fourier, $10 \%$ oversampling), 3296 radial angles, 12 min scan time. $P_{2}(\mathrm{~m} / 79 \mathrm{y} / 96 \mathrm{~kg})$ and $P_{3}(\mathrm{~m} / 67 \mathrm{y} / 115 \mathrm{~kg})$ : sagittal slab orientation $(\mathrm{FOV} 400 \times$ 
$\left.400 \times 360 \mathrm{~mm}^{3}\right)$, spatial resolution $1.65 \times 1.65 \times 5 \mathrm{~mm}^{3}, 256$ pixel matrix, 72 slices (61\% slice res., 5/8 partial Fourier), 4416 radial angles, 10 min scan time.

To demonstrate the applicability of the derived motion model, $N_{\text {Bins }}=5$ respiratory states were used. MR data were reconstructed using regridding. The empirically selected parameters for the deformable registration were smoothing $\alpha=5,75 \%$ randomized sampling, and 3 levels with a grid spacing of 8,4 , and 2 . The PET reconstruction (OSEM3D 3i21s) used 3 iterations on 21 subsets, with a matrix size of $172 \times 172$ and $4 \mathrm{~mm}$ Gaussian post-reconstruction filtering.

\section{Results and Discussion}

\section{$5.1 \quad$ Self-gating}

In all cases, the self-gating signal was successfully extracted. Fig. 2a) shows the detected maximal edge displacement depending on the number of bins chosen. An asymptotic behavior, caused by successive reduction of intra-bin motion with a higher number of bins [19], was observed. The maximal displacement in the three patients was $10-13 \mathrm{~mm}$. Due to the uniform bin size, a relatively high number of bins is required to capture the full respiratory range, eventually compromising image quality in the exhale bins. A cumulative respiratory histogram was computed from the detected liver edge displacements using $N_{\text {Bins }}=15$. As can be seen in Fig. 2b), 60-75\% of the detected respiration was in a range of 5 mm from maximal end-expiration, while $\sim 20 \%$ are within $5 \mathrm{~mm}$ from maximal inspiration. Thus, when using only 5 bins, the intra-bin motion blur causes the edge of the last bin to be detected at about 2-3 $\mathrm{mm}$ below maximal inspiration (cf. also Fig. $2 \mathrm{a}$ )). The choice of $N_{\text {Bins }}=5$ can be considered a compromise between accuracy and sufficient statistics for all bins.

Upon comparing the motion model with the Dixon images acquired for the $\mu$-map it was noticed that $P_{2}$ and $P_{3}$ had held their breath at end-inspiration rather than at end-expiration as instructed. Therefore, in both cases the deformed $\mu$-maps $\hat{M}_{i}$ were generated by applying the model $\boldsymbol{T}_{5, i}$. Moreover, the end-exhale $\mu$-map for reconstructions $R_{\mathrm{All}}, R_{40}$ was estimated by appling $\boldsymbol{T}_{5,1}$.
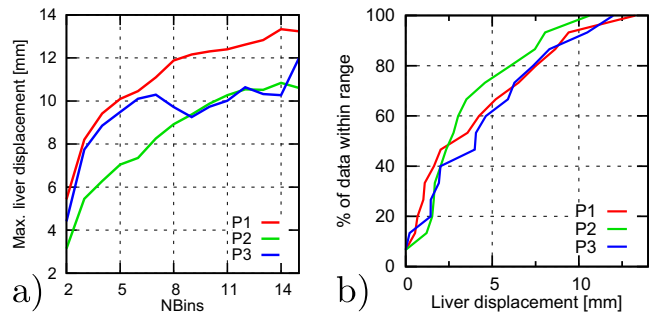

Fig. 2. a) Maximal displacement of liver edge depending on $N_{\text {Bins. }}$ b) Cumulative respiratory histogram for $P_{1}-P_{3}$.

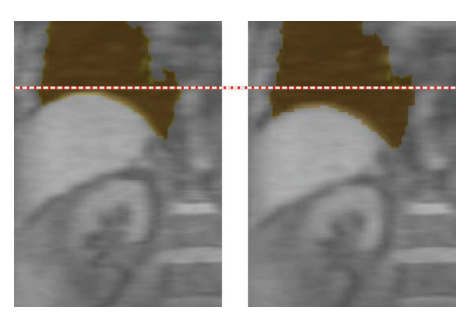

Fig. 3. Self-gated MRI of $P_{3}$ overlaid with warped $\mu$-map (brown: lung compartment) 


\subsection{PET Reconstruction}

The tissue interfaces in the deformed $\mu$-maps were visually consistent with the acquired motion model, as shown in Fig. 3 depicting a fusion of the motion model at end-expiration and end-inspiration with the corresponding $\mu$-maps in $P_{3}$.

In $P_{1}$, a lesion $(\varnothing 20 \mathrm{~mm})$ was found to move by approx. $6 \mathrm{~mm}$ in head-feet direction in the individual gated PET reconstructions of $G_{5}$. Fig. 4 shows a line profile through the lesion, averaged over $3 \times 3$ pixels. In the ungated reconstruction, the lesion appears elongated by $\sim 5 \mathrm{~mm}$. As shown in Fig. 4 $\mathrm{b}$ ), the true shape was restored after post-reconstruction registration. Corresponding PET images are given in Fig. [5a).

In $P_{2}$, a larger lesion (Ø $45 \mathrm{~mm}$ ) was found in the liver. Significant respiratory movement in the anteriorposterior direction was visible in the model and confirmed by the direction of motion blur in the ungated $\mathrm{PET}$ reconstruction. The $\mathrm{SUV}=6$ iso-intensity contour of the lesion for all reconstructions is shown in Fig. [5b), as an overlay in the end-exhale motion model. Gated and corrected reconstructions match the outlines of the slightly hypointense region in the MR image well, while the ungated contour is displaced by $\sim 6 \mathrm{~mm}$.
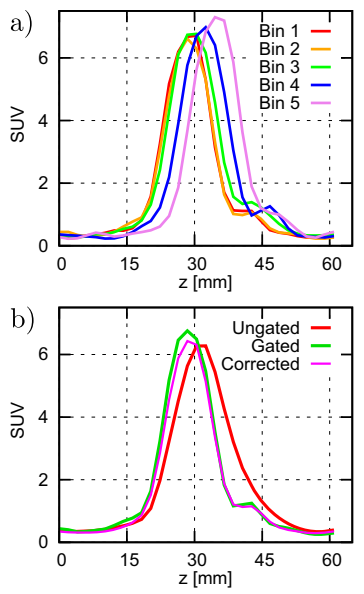

Fig. 4. Line profiles through pulmonary lesion in $P_{1}$

In $P_{3}$, multiple foci ( $\varnothing$ up to $10 \mathrm{~mm}$ ) in both kidney cortices showed increased uptake of FDG. The largest spot was considered pathological. Due to respiratory motion, the foci were less conspicuous in the ungated images, cf. Fig. 55).

The lesions were segmented with fixed thresholds of SUV=2.5 $\left(P_{1}\right)$ and $\mathrm{SUV}=6\left(P_{2}, P_{3}\right)$ and the apparent lesion volume was compared against the gated reconstruction $R_{40}$. For $\left(P_{1}, P_{2}, P_{3}\right)$, the difference was reduced from $(+27.5 \%$, $+18.2 \%,-58.9 \%)$ in the ungated reconstructions, respectively, to $(+1.1 \%,+6.4 \%$, $+15.3 \%$ ) by applying the proposed method. Loss of contrast in the motioncompensated reconstruction, as also reported by [4], was noticed for few very small details. A possible reason is residual intra-bin motion especially in endinspiration, as discussed in Section 5.1. Moreover, $R_{40}$ utilizes $40 \%$ of the data for reconstruction, while the bins of $G_{5}$ are reconstructed from only $20 \%$. The non-linear reconstruction may recover such small structures in the former case, but fail to do so in the latter. Here, applying the motion compensation already during rather than after PET reconstruction [12] promises improved sensitivity.

\section{Summary and Conclusion}

We presented first in-vivo results of self-gated MRI motion modeling applied to respiratory motion compensation in $\mathrm{PET} / \mathrm{MR}$ scanners. It requires no additional physiological signal sensors and captures an averaged respiratory motion cycle throughout the measurement. This information is used to correct for respiratory 

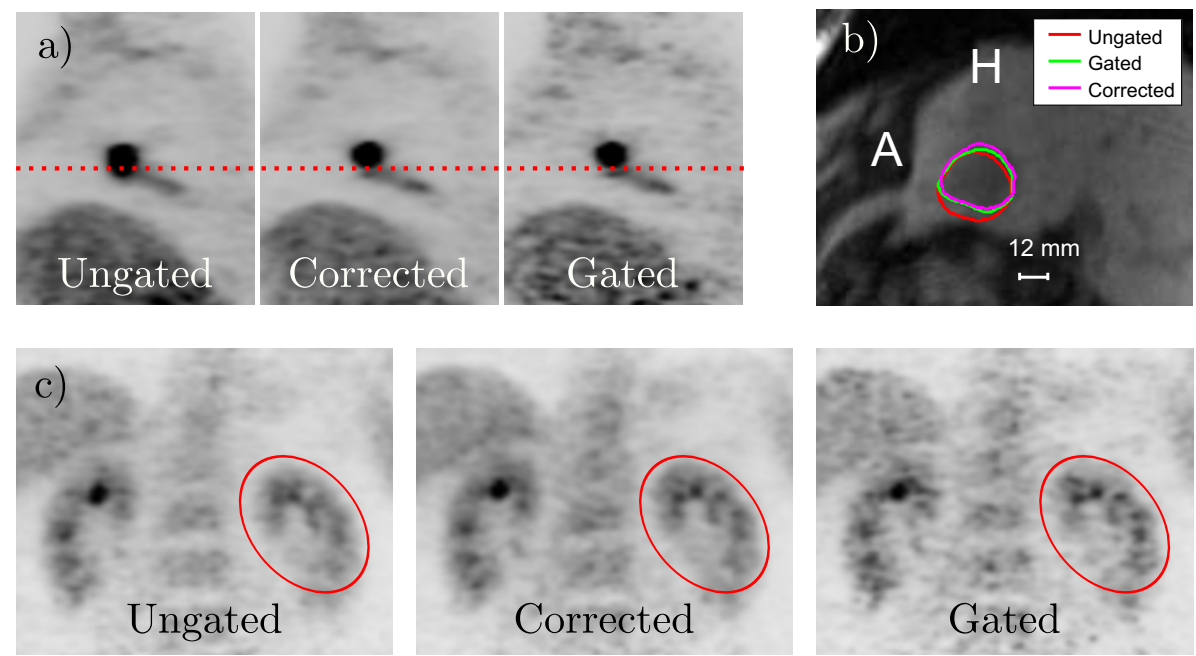

Fig. 5. a) Sagittal slice through pulmonary lesion in $P_{1}$. b) SUV=6 iso-contours as overlay over MR image of liver lesion in $P_{2}$. c) Spots in left kidney cortex in $P_{3}$ are more clearly enhanced in the motion-compensated and gated reconstructions.

motion in the PET reconstruction, resulting in a clear reduction of motion blur but improved SNR compared to conventional gated reconstructions. The approach was validated on clinical patients with lesions in three different organs.

Further investigations will consider applying the motion model in motioncompensation incorporated PET reconstructions [12], especially utilizing a higher number of respiratory bins. Moreover, despites the advantage of capturing the global course of respiration during the examination, the scan time for motion modeling is relatively long. A reduction is easily possible by acquiring fewer radial spokes at the cost of increased radial streaking artifacts. Robust registration methods on the one hand and Compressed Sensing reconstruction enforcing a temporal regularization on the MRI images on the other hand can help to overcome the undersampling artifacts. Finally, the proposed method will be extended to automatically detect the actual respiratory position of the acquired $\mu$-map.

\section{References}

1. Keller, S.H., Holm, S., Hansen, A.E., Sattler, B., Andersen, F., Klausen, T.L., Højgaard, L., Kjær, A., Beyer, T.: Image artifacts from MR-based attenuation correction in clinical, whole-body PET/MRI. MAGMA 26, 173-181 (2013)

2. Liu, C., Pierce, L.A., Alessio, A.M., Kinahan, P.E.: The impact of respiratory motion on tumor quantification and delineation in static PET/CT imaging. Phys. Med. Biol. 54(24), 7345-7362 (2009)

3. Bundschuh, R.A., Martínez-Möller, A., Essler, M., Nekolla, S.G., Ziegler, S.I., Schwaiger, M.: Local motion correction for lung tumours in PET/CT-first results. Eur. J. Nucl. Med. Mol. Imaging 35(11), 1981-1988 (2008) 
4. Würslin, C., Schmidt, H., Martirosian, P., Brendle, C., Boss, A., Schwenzer, N.F., Stegger, L.: Respiratory motion correction in oncologic PET using T1-weighted MR imaging on a simultaneous whole-body PET/MR system. J. Nucl. Med. (2013)

5. McClelland, J.R., Hawkes, D.J., Schaeffter, T., King, A.P.: Respiratory motion models: A review. Med. Image Anal. 17(1), 19-42 (2013)

6. Buerger, C., Tsoumpas, C., Aitken, A., King, A.P., Schleyer, P., Schulz, V., Marsden, P.K., Schaeffter, T.: Investigation of MR-based attenuation correction and motion compensation for hybrid PET/MR. IEEE Trans. Nucl. Sci. 59(5), 1967$1976(2012)$

7. Dikaios, N., Izquierdo-Garcia, D., Graves, M.J., Mani, V., Fayad, Z.A., Fryer, T.D.: MRI-based motion correction of thoracic PET: initial comparison of acquisition protocols and correction strategies suitable for simultaneous PET/MRI systems. Eur. Radiol. 22(2), 439-446 (2012)

8. King, A.P., Buerger, C., Tsoumpas, C., Marsden, P.K., Schaeffter, T.: Thoracic respiratory motion estimation from MRI using a statistical model and a 2-D image navigator. Med. Image Anal. 16(1), 252-264 (2012)

9. Ouyang, J., Li, Q., El Fakhri, G.: Magnetic resonance-based motion correction for positron emission tomography imaging. Semin. Nucl. Med. 43(1), 60-67 (2013)

10. Guérin, B., Cho, S., Chun, S.Y., Zhu, X., Alpert, N.M., El Fakhri, G., Reese, T., Catana, C.: Nonrigid PET motion compensation in the lower abdomen using simultaneous tagged-MRI and PET imaging. Med. Phys. 38(6), 3025-3038 (2011)

11. Chun, S.Y., Reese, T.G., Ouyang, J., Guérin, B., Catana, C., Zhu, X., Alpert, N.M., El Fakhri, G.: MRI-based nonrigid motion correction in simultaneous PET/MRI. J. Nucl. Med. 53(8), 1284-1291 (2012)

12. Lamare, F., Ledesma Carbayo, M.J., Cresson, T., Kontaxakis, G., Santos, A., Le Rest, C.C., Reader, A.J., Visvikis, D.: List-mode-based reconstruction for respiratory motion correction in PET using non-rigid body transformations. Phys. Med. Biol. 52(17), 5187-5204 (2007)

13. Polycarpou, I., Tsoumpas, C., Marsden, P.K.: Analysis and comparison of two methods for motion correction in PET imaging. Med. Phys. 39(10), 6474-6483 (2012)

14. Chun, S.Y., Fessler, J.A.: Spatial resolution properties of motion-compensated tomographic image reconstruction methods. IEEE Trans. Med. Imaging 31(7), 14131425 (2012)

15. Rahmim, A., Tang, J., Zaidi, H.: Four-dimensional image reconstruction strategies in cardiac-gated and respiratory-gated PET imaging. PET Clinics 8(1), 51-67 (2013)

16. Winkelmann, S., Schaeffter, T., Koehler, T., Eggers, H., Doessel, O.: An optimal radial profile order based on the golden ratio for time-resolved MRI. IEEE Trans. Med. Imaging 26(1), 68-76 (2007)

17. Grimm, R., Bauer, S., Kiefer, B., Hornegger, J., Block, T.: Optimal channel selection for respiratory self-gating signals. In: Proc. 21st Annual Meeting ISMRM, Salt Lake City, Utah, USA, p. 3749 (2013)

18. Heinrich, M.P., Jenkinson, M., Brady, S.M., Schnabel, J.A.: Globally optimal deformable registration on a minimum spanning tree using dense displacement sampling. In: Ayache, N., Delingette, H., Golland, P., Mori, K. (eds.) MICCAI 2012, Part III. LNCS, vol. 7512, pp. 115-122. Springer, Heidelberg (2012)

19. Dawood, M., Büther, F., Stegger, L., Jiang, X., Schober, O., Schäfers, M., Schäfers, K.P.: Optimal number of respiratory gates in positron emission tomography: a cardiac patient study. Med. Phys. 36(5), 1775-1784 (2009) 